Themenheft Nr. 40: CoViD-19 und die digitale Hochschulbildung. Irritationen, Einsichten und Programmatiken

Herausgegeben von Markus Deimann, Marios Karapanos und Klaus Rummler

\title{
Akademische Mediennutzung Studierender im Corona-Semester 2020
}

\section{Digitalisierungsschub oder weiter wie bisher?}

Svenja Bedenlier ${ }^{1}$, Marion Händel ${ }^{1}$ (D), Rudolf Kammerl ${ }^{1}$ (D), Michaela Gläser-Zikuda ${ }^{1}$ (D), Bärbel Kopp ${ }^{1}$ und Albert Ziegler ${ }^{1}$ (D)

${ }^{1}$ Friedrich-Alexander-Universität Erlangen-Nürnberg

\begin{abstract}
Zusammenfassung
Unter dem Eindruck der CoViD-19 Pandemie erfolgte im Sommersemester 2020 an deutschen Hochschulen flächendeckende Online-Lehre im Notbetrieb. Die vorliegende längsschnittliche Fragebogenstudie untersucht die akademische Mediennutzung, -kompetenz und-ausstattung Studierender ( $N=2.037$ ) an einer deutschen Volluniversität zu Beginn und zur Mitte des betreffenden Semesters. Die Ergebnisse zeigen, dass die akademische Mediennutzung intensiviert wird, wahrgenommene digitale Kompetenzen der Studierenden zunehmen und E-Learning Angebote fakultätsspezifisch eingesetzt und genutzt werden. Sichtbar wird jedoch auch, dass Studierende mit heterogenen Voraussetzungen in das Semester starteten, die sich auch im Semesterverlauf auf die Studiensituation auswirkten. Die Ergebnisse werden vor dem Hintergrund bildungspolitischer Bestrebungen hinsichtlich der Digitalisierung der Hochschullehre, mediendidaktischer Fragestellungen und studentischem Eigensinn der Mediennutzung eingeordnet und diskutiert.
\end{abstract}

Students' Academic Media Use in the Corona Semester 2020. Digitization Boost or Continuing as Usual?

\begin{abstract}
Against the backdrop of the CoViD-19 pandemic, German higher education switched to full online teaching in the summer term 2020. The current longitudinal study based on a questionnaire investigates students' $(N=2,037)$ academic media usage, competence and equipment at one German comprehensive university prior and during that specific term. Results show that academic media usage intensifies, perceived digital competencies increase and that provision and usage of e-learning tools occurs differently across faculties. It is also indicated that students start into the term with heterogeneous prerequisites, also affecting their study situation as the summer term evolves. The study results are discussed
\end{abstract}


against the background of education policy on digitization of higher education teaching and learning, questions of designing technology-enhanced learning and students' own sense making of media usage in higher education.

\section{Einleitung}

Digitale Medien und digital vermittelte Lehr-Lern-Formate sind heute - in unterschiedlichem Ausmass - fester Bestand der Hochschullehre sowie Gegenstand bildungspolitischer Forderung und Förderung (Gilch et al. 2019; Kultusministerkonferenz 2019). Bedingt durch die CoViD-19 Pandemie und dem emergency remote teaching (Hodges et al. 2020) als pragmatischer Antwort, erfolgte an deutschen Hochschulen im Sommersemester 2020 erstmalig flächendeckende, reine Online-Lehre. Diese wurde, mit Fokus auf bspw. Veranstaltungsformate und eingesetzte Lerntechnologien (Skulmowski und Rey 2020), den sozio-emotionalen Zustand Studierender (Händel, Stephan et al. 2020) oder die Erwartungen und Einstellungen Lehrender gegenüber der unerwarteten Online-Lehre (Daumiller et al. 2021) breitflächig untersucht. Ebenso führten zahlreiche Hochschulen Evaluationsstudien innerhalb der Institutionen durch, um Einblicke in verschiedene lehr-lernbezogene Dimensionen zu erhalten (Arndt, Ladwig, und Knutzen 2020). Studierende und die Analyse ihrer (studienbezogenen) Situation stehen im Fokus von 52 der 69 Studien, die im Review von Arndt, Ladwig und Knutzen (2020) berücksichtigt werden, und die hochschulübergreifend Aufschluss über Themen wie studentische Vorerfahrungen mit digitalen Medien in der Lehre, wahrgenommene Kompetenzen und technische Ausstattung im Sommersemester 2020 geben.

Während die CoViD-19 bedingte Ausnahmesituation einerseits als «Digitalisierungsschub» (Winde et al. 2020, 2) für den Bildungsbereich Hochschule gewertet wird, so wurde gleichermassen ein offener Brief mit über 15.000 Unterzeichnenden verfasst, der ein Nichtsemester des Sommersemesters 2020 im Sinne der Studierenden forderte («Das Sommersemester muss...» 2020) - dies nicht zuletzt mit dem Argument, dass Lehrende und Studierende vielfach nicht ausreichend «mit den Methoden und Tools des E-Learning» (ebd.) vertraut seien.

Die Nutzung von digitalen Medien, bzw. Bildungs- oder Lerntechnologien im Studium und der kompetente Umgang mit innen, ist eine Erwartung, die bereits vor dem Ausbruch der Pandemie an Studierende gestellt wurde und dies sowohl unter einer bildungspolitischen (KMK 2019) als auch unter einer stärker individuumszentrierten Sichtweise (Senkbeil, Ihme, und Schöber 2019). So thematisieren verschiedene quantitative Untersuchungen studentische Mediennutzung im akademischen Kontext (für eine Übersicht: Steffens, Schmitt, und Assmann 2017). Diese berücksichtigten die technische Ausstattung von Studierenden, identifizierten Nutzungscluster und soziodemografische Unterschiede zwischen Studierendengruppen sowie 
Nutzungshäufigkeiten spezifischer (institutionell) bereitgestellter digitaler Werkzeuge über die Zeit (z. B. Dolch und Zawacki-Richter 2018; Gidion und Grosch 2011; Persike und Friedrich 2016; Zawacki-Richter 2015; Zawacki-Richter, Kramer und Müskens 2016). Ebenso wurden studentische Kompetenzen erfassbar gemacht und untersucht (Hong und Kim 2018; Senkbeil, Ihme, und Schöber 2019, 2020).

Bedingt durch die pandemische Situation und ihrem Einfluss auf die Gestaltung von Lehre und daraus resultierenden Anforderungen an die Beteiligten seit Anfang 2020 ist zu erwarten, dass sich studentische Mediennutzung und -kompetenz verändert und sich studentische Medienausstattung an diese Bedingungen angepasst haben.

\section{Theoretischer Hintergrund}

\subsection{Digitalisierung in der Hochschullehre}

Digitalisierung, digitale Medien und digitale Bildung gewinnen in der bildungspolitischen Diskussion bereichsübergreifend als Begriffe an Bedeutung und werden nicht zuletzt als Mittel einer «Weiterentwicklung der Lehre» (KMK 2019, 8) und als Teil der "strategischen Gesamtentwicklung» (KMK 2019, 7) von Hochschulen beschrieben. Einzelne Bundesländer haben in den letzten Jahren Digitalisierungsstrategien für ihren Bildungs- bzw. Hochschulbereich verabschiedet (so z. B. Sachsen, Bayern und Thüringen; Gilch et al. 2019); Der Einsatz digitaler Medien für die Hochschullehre ist strategisch konnotiert mit den Begriffen der Qualität und Effizienz, welche über diese beibehalten und gesteigert werden soll (Bedenlier und Deimann 2020). Mit der Einrichtung des Hochschulforums Digitalisierung wurde dem Aspekt der Digitalisierung vor allem im Lehr-Lern-Kontext eine Diskussionsplattform geschaffen, die Akteure aus Praxis, Forschung und Politik zusammenbringt und anwendungsbezogene Impulse für eine Digitalisierung an Hochschulen generiert (z. B. Hochschulforum Digitalisierung 2015). Analog zur Diskussion der verschiedenartigen Verwendungen des Begriffs Medienbildung (Jörissen 2011; Tulodziecki 2011), kann so für die oben genannten Kontexte eine «bildungspolitische, administrative Perspektive» (Jörissen 2011, 213) angenommen werden, die weniger individuelle Praktiken, Erfahrungen und konzeptionell-theoretische Auseinandersetzung mit digitalen Medien im Hochschulkontext fokussiert (siehe Hofhues et al. 2020 für einen derartigen Ansatz).

Gleichwohl stehen Studierende als Zielgruppe der (digitalen) Hochschullehre im besonderen Fokus dieser öffentlichen Diskussion, exemplarisch dargestellt über die Zielsetzung der Ausbildung studentischer digitaler Kompetenz und Medienkompetenz in den Fächern (HRK 2019), ebenso wie in den Digitalisierungsstrategien für die Lehre an verschiedenen Hochschulen (z. B. RUB o. D.; RWTH Aachen o. D.) oder 
als Zielgruppe und Beteiligte an aktuell aufgeworfenen Positionsbestimmungen wie «New Learning» (FernUniversität Hagen 2020). Folgt man dem Monitor Digitale Bildung (Schmid et al. 2017), so sind Studierende weniger treibende Kräfte für die Digitalisierung der hochschulischen Lehre, sie sehen sich allerdings durch diese in einigen Disziplinen stärker zum Lernen motiviert. Weitere individuelle und soziale sowie kontextbezogene Merkmale bei den Studierenden werden als Voraussetzung für digitale Lehre untersucht.

\subsection{Mediennutzung, technische Ausstattung und Medienkompetenz Studierender}

Studentische Nutzung von Medien, E-Learning Tools und technische Ausstattung Die Untersuchung studentischer Mediennutzung erfolgte im deutschsprachigen Kontext bislang über primär quantitative Zugänge, die vor allem die Nutzungshäufigkeit bestimmter digitaler Medien und Formate im Studienkontext in den Blick nahm (z. B. Bond et al. 2018; Dolch und Zawacki-Richter 2018; Gidion und Weyrich 2017; Kreidl und Dittler 2018), darüber unterschiedliche Gruppen von Nutzern und Nutzerinnen identifizierte (Persike und Friedrich 2016) oder Verläufe der Nutzung über die Zeit dokumentierten (Zawacki-Richter, Kramer, und Müskens 2016). Unterschiede in der Nutzung zeigen sich so beispielweise zwischen sogenannten traditionellen und nicht-traditionellen Studierenden hinsichtlich der Wahrnehmung der Nützlichkeit digitaler Medien für das Studium (Zawacki-Richter 2015) oder zwischen Studierenden der Cluster digitale Allrounder und PDF-Nutzende (Persike und Friedrich 2016) in Bezug auf die Vielfalt und Häufigkeit der genutzten Medien. Die studentische Ausstattung mit studienrelevanter Hardware und Verfügbarkeit über einen Internetzugang werden in Studien zum Mediennutzungsverhalten oftmals berücksichtigt. So zeigt beispielsweise die Studie von Kreidl und Dittler (2018) mit 4.000 Studierenden, dass diese zu 96 \% ein Smartphone und zu 95 \% einen Laptop o. ä. Gerät besitzen. Zugang zum Internet haben rund $99 \%$ der Studierenden in drei Befragungskohorten einer grösseren Mediennutzungsstudie, in 2018 haben $98 \%$ der Befragten auch mobiles Internet und berichten für diesen Zeitpunkt ebenso zu rund $95 \%$ einen Laptop oder Smartphone zu besitzen (Zawacki-Richter 2020). Auch zu Beginn des Sommersemesters 2020 berichten die Studierenden überwiegend, technisch ausreichend ausgestattet zu sein, wobei spezifisches Equipment zugekauft werden musste (Arndt et al. 2020).

Primärstudien und Evaluationen im Kontext der Umstellung auf emergency remote teaching (Hodges et al. 2020) weisen zu studentischer Medien- und E-Learning Tool Nutzung verschiedene Befunde auf, die vergleichbar sind mit denen der oben angeführten Studien: In ihrer Befragung von 3.469 Studierenden verschiedener 
Studienfächer zum digitalen Semester an sächsischen Hochschulen zeigen Karapanos, Pelz, Hawlitschek und Wollersheim (2021) unter anderem, dass Studierende weitestgehend mit Hardware ausgestattet sind, jedoch nur $76 \%$ der Befragten über einen stabilen Internetzugang verfügen. Die von den Studierenden genutzten E-Learning Tools waren in den Veranstaltungen vor allem Lernmanagementsysteme, textbasierte Formate (Foren und Literatur) und Videokonferenzen. Im Unterschied dazu konstatieren Mulders und Krah (2021) in ihrer Untersuchung der Akzeptanz von ELearning bei Bachelorstudierenden der Erziehungswissenschaft im Sommersemester 2020, dass rund $90 \%$ der Befragten für die Online-Lehre ausreichend technisch ausgestattet waren und, dass sozioökonomische Charakteristika mit Akzeptanz- und Nutzungswerten kaum in Zusammenhang stehen.

In den oben angeführten Untersuchungen vor 2020, aber auch denjenigen, die Arndt et al. (2020) im Kontext der pandemischen Situation zusammenfassend analysieren, wird ein Fokus auf die deskriptive Darstellung des jeweiligen Nutzungsverhaltens gelegt. Eine tiefergehende Betrachtung studentischer Sichtweisen und Analyse ihrer medialen Praktiken im Studium werden nicht durchgeführt (Hofhues et al. 2020).

\section{Studentische Medienkompetenz}

Hinsichtlich studentischer Mediennutzung treten nicht zuletzt auch Fragen der dafür notwendigen Kompetenz und grundsätzlichen Zugangsmöglichkeiten zu technischer Infrastruktur und Internet auf. Dabei werden in der Forschung die Kompetenzen sehr unterschiedlich fokussiert und operationalisiert. Während Studien im Schulbereich auf die Unterschiede in computer- und informationsbezogenen Kompetenzen in Abhängigkeit sozioökonomischer Merkmale hinweisen (Vennemann et al. 2019) und digitale Ungleichheit diskutiert werden (Kutscher 2019; Verständig, Klein, und Iske 2016), so kann diese Diskussion für den Hochschulbereich noch intensiviert werden (z. B. Schmölz, Geppert, und Barberi 2020). Akademische Medienkompetenz ist trotz des Narratives der digital natives (Prensky 2001) nicht voraussetzbar (Lei 2009) und ist zudem, wie Senkbeil, Ihme und Schöber (2019) zeigen, innerhalb verschiedener Studierendengruppen unterschiedlich stark ausgeprägt - so beispielsweise zugunsten männlicher Studierender, bestimmter Fächergruppen und Studierender fortgeschrittener Semester. Besonders für Studierende des Lehramts zeigen Senkbeil, Ihme und Schöber (2020), dass deren ICT Literacy geringer ausgeprägt ist als die von Studierenden anderer Fachrichtungen und sie vielfach auch während des Studiums ein entsprechendes Mindestmass nicht erreichen. Während beispielsweise die Studien von Senkbeil und Kollegen $(2019,2020)$ vor allem die kompetente Nutzung digitaler Anwendungen für studienbezogene Zwecke fokussieren und auch die in der vorliegenden Studie eingesetzte Skala zur Erfassung der digital readiness for academic 
engagement (DRAE; Hong und Kim, 2018) auf einen sehr spezifischen Aspekt von Medienkompetenz ausgerichtet ist, so werden Fragen der Medienkritik oder Reflexion ausgespart (im Unterschied z. B. zu dem DigComp 2.1 Framework von Carretero, Vuokari, und Punie 2017).

Die Situation im Sommersemester 2020 stellte Lehrende und Studierende vor die Herausforderung, sich auf ein vollständiges Online-Semester einzulassen - so dass aus einer pragmatischen Sicht heraus die kompetente Nutzung, im Sinne von DRAE, im Vordergrund stand. Interessanterweise bewerteten die befragten Studierenden in der Studie von Winde et al. (2020) die mangelnde Kompetenz mit $4 \%$ als kleinste Herausforderung - mangelndes Sozialleben und Motivations- und Konzentrationsproblems (69 \% bzw. 59 \%) lagen hingegen auf den beiden vorderen Plätzen. Gleichwohl zeigt die Studie von Muilenburg und Berge (2005), dass Lernende, die im Umgang mit online learning technology sicher sind, unter anderem signifikant weniger Barrieren empfinden für Bereiche wie soziale Interaktion und Umgang mit Lehrenden, aber dafür eine höhere Lernmotivation aufweisen. Digitale Kompetenzen zeigen sich hier also eher implizit und ihr Fehlen spielt im Kontext des digitalen Sommersemesters möglicherweise in die wahrgenommenen Motivationsschwierigkeiten hinein.

\section{Fragestellung}

Die Intention dieses Beitrags ist die Analyse und kritische Reflexion studentischer Medienausstattung, -nutzung und -kompetenz, sowie erste Schlussfolgerungen für die theoretische und praktische Diskussion zur zukünftigen Gestaltung hochschulischer Lehre zu formulieren. Es werden drei zentrale Fragestellungen untersucht:

- Wie starten Studierende in das erste Digitalsemester? Das heisst, wie gut sind sie ausgestattet, welches Mediennutzungsverhalten zeigen sie und wie schätzen sie selbst ihre akademische Medienkompetenz ein? (F1)

- Wie verändern sich Mediennutzung, Nutzung von E-Learning Tools und Medienkompetenz der Studierenden im Verlauf des Semesters? (F2)

- Unterscheidet sich die Nutzung von E-Learning Angeboten im Semester in Abhängigkeit bestimmter Studierendengruppen (indiv. Merkmale, wie Geschlecht, angestrebter Studienabschluss, Migrationshintergrund und strukturelle Merkmale, wie Fakultätszugehörigkeit)?(F3) 


\section{Methode}

\subsection{Studiendesign}

An einer deutschen Volluniversität wurde im Sommersemester 2020 eine längsschnittliche quantitative Erhebung durchgeführt. Zu drei Messzeitpunkten - vor, während und nach Ende der Vorlesungszeit - wurden Studierende in einem längsschnittlichen Personenmatching unter anderem zu ihrem Mediennutzungs- und Lernverhalten befragt. Die Datenerhebung erfolgte anhand von Online-Befragungen über Unipark Questback EFS (unipark.com).

Im Fokus dieses Beitrags stehen die Ergebnisse des ersten und zweiten Messzeitpunkts, die die Nutzung von (Lern-)Medien im Vergleich von pre-CoViD-19 und IstZustand während des Corona-Semesters erlauben.

\subsection{Stichprobe}

Alle Studierenden einer Universität mit insgesamt rund 38.500 Studierenden wurden via Mail einmalig pro Erhebungszeitpunkt zur Studienteilnahme eingeladen. Nach Zustimmung zur Studienteilnahme wurden unter Einhaltung der Datenschutzrichtlinien alle erhobenen Daten pseudonymisiert. Den Teilnehmenden entstanden durch die (Nicht-)Teilnahme keinerlei Vor- oder Nachteile.

Über beide Messzeitpunkte hinweg nahmen $N=2.037$ Studierende teil. Die Studierenden waren durchschnittlich 23.0 Jahre alt (SD = 4.4). 59.0\% der Studierenden waren weiblich, $29.6 \%$ männlich, $0.1 \%$ divers und $11.3 \%$ machten keine Angabe zum Geschlecht. Studierende aller Fakultäten und angestrebter Studienabschlüsse nahmen an den Befragungen teil (vgl. Tabelle 1). Die unterschiedlichen Rücklaufquoten pro Fakultät entsprechen den Studierendenzahlen pro Fakultät.

\begin{tabular}{|l|c|}
\hline Fakultät & N \\
\hline Philosophische Fakultät und Fachbereich Theologie & 577 \\
\hline Naturwissenschaftliche Fakultät & 283 \\
\hline Rechts- und Wirtschaftswissenschaftliche Fakultät & 404 \\
\hline Technische Fakultät & 431 \\
\hline Medizinische Fakultät & 341 \\
\hline
\end{tabular}

Tab. 1.: Studienteilnehmende pro Fakultät.

Jeweils $36 \%$ der Studierenden strebten entweder einen Bachelorabschluss oder einen Staatsexamensabschluss an, $24.1 \%$ einen Masterabschluss, $1.3 \%$ eine Promotion und $1.6 \%$ einen anderen Abschluss. 
Zur familiären Situation gaben 3.6 \% der teilnehmenden Studierenden an, Aufgaben in der Kinderbetreuung im eigenen Haushalt erfüllen zu müssen (ein oder mehrere Kinder im Haushalt). Schliesslich wiesen $10.5 \%$ der Studierenden einen Migrationshintergrund auf (nicht-deutsches Geburtsland und/oder nicht-deutsche Erstsprache).

\subsection{Messinstrumente}

Im Rahmen der Studie wurde jeweils zu beiden Erhebungszeitpunkten mit überwiegend geprüften Instrumenten (a) die Arbeitsplatzausstattung, (b) die Nutzung von Arbeitsgeräten, (c) die selbstwahrgenommenen Kompetenzen für den Umgang mit digitalen Medien und (d) die Nutzung von an der Universität angebotenen E-Learning Tools erfragt.

Die Arbeitsplatzausstattung wurde im Ja/Nein-Format erfragt, d. h. ob die Studierenden Zugang haben zu einem ruhigen Arbeitsplatz sowie mindestens einem Endgerät (stationärer Desktop-PC, Notebook/Laptop oder Tablet-PC). Sofern die Geräte verfügbar waren, wurde die Nutzung anhand einer 5-stufigen Likert-Skala abgefragt - (fast) nie, 1-2 Mal/Monat, 1-2 Mal/Woche, fast jeden Tag, täglich.

Neben Ausstattungsmerkmalen wurden ausserdem die selbsteingeschätzten Kompetenzen im Umgang mit digitalen Medien erfasst. Hierzu wurden insgesamt acht Items aus zwei Subskalen zum Umgang mit Hardware und dem Teilen digitaler Informationen des DRAE (Hong und Kim 2018) eingesetzt. Die Items waren auf einer sechsstufigen Likert-Skala zu beantworten (stimmt gar nicht bis stimmt genau). Die interne Konsistenz war zu beiden Messzeitpunkten zufriedenstellend (Cronbachs $\alpha=.86 / .85$; Beispielitem: «Ich kann Medien, digitale Fotos, Dateien, Videodateien und Audiofiles hoch- und runterladen.»).

Die Nutzung von E-Learning Tools wurde in Anlehnung an den jährlich stattfindenden Studierenden-Survey der Universität (Froebus und Bender 2019) für folgende Angebote im Ja/Nein- Format erfasst: Live-Streams von Vorlesungen, Vorlesungsaufzeichnungen Herunterladbare Vorlesungsskripte/Literatur, Online-Lernmodule, Einsatz von digitalen Medien in Lehrveranstaltungen (z. B. Live-Voting), OnlineSelbsttests zur Selbstkontrolle, Online-Kommunikation und Zusammenarbeit, und andere online-gestützte Lernangebote. Die Antwortoption des bisherigen Nichtwahrnehmens von Angeboten wurde ebenso gegeben.

\subsection{Datenauswertung}

Um die Medienausstattung sowie die Medien und E-Learning Tool Nutzung vor und während des ersten Corona-Semesters zu untersuchen (F1), wurden deskriptive Statistiken berechnet. Potentielle Ausgangsunterschiede hinsichtlich der technischen 
Ausstattung sowie der selbstwahrgenommenen Kompetenzen zwischen bestimmten Studierendengruppen (Geschlecht, Studienfach, Fakultätszugehörigkeit, Studierende mit Kind, Studierende mit Migrationshintergrund) wurden anhand multivariater Varianzanalysen getestet.

Veränderungen in den untersuchten Variablen wurden anhand gepaarter $t$-tests analysiert (F2). Schliesslich wurden anhand multivariater Varianzanalysen Nutzungsunterschiede der E-Learning Angebote im Corona-Semester zwischen Studierendengruppen geprüft (F3). Alle Analysen wurden mit SPSS, Version 26 vorgenommen.

\section{Ergebnisse}

\subsection{Ausgangslage}

Die in Tabelle 2 dargestellten Ergebnisse zeigen, dass die meisten Studierenden vor Semesterstart gut ausgestattet sind, d. h. mehrheitlich über einen ruhigen Arbeitsplatz verfügen und die Befragten bereits vor Start des Corona-Semesters über mindestens ein studienrelevantes Endgerät verfügen (z. B. Desktop-PC, Tablet oder Notebook).

Im Durchschnitt gaben die Befragten an, dieses Endgerät fast täglich bis mehrmals täglich zu nutzen. Eine deskriptiv höhere Nutzung zeigte sich bei der Smartphone-Nutzung sowie der Internetnutzung. Im Umgang mit Endgeräten sowie dem Teilen digitaler Informationen fühlten sich die befragten Studierenden kompetent.

Bedeutsame Unterschiede zeigten sich zwischen verschiedenen Studierendengruppen. Eine multivariate Varianzanalyse mit Geschlecht, Fakultätszugehörigkeit, angestrebtem Studienabschluss, zu betreuenden Kindern, und Migrationshintergrund als unabhängige Variablen und drei abhängigen Variablen (Ausstattung mit ruhigem Arbeitsplatz, digitalem Endgerät, Kompetenzen im Umgang mit Soft- und Hardware) erbrachte signifikante Effekte für die untersuchten Variablen (Geschlecht: Wilks $\lambda=.910, F(3,1800)=59.66, p<.001, \eta^{2}=.09$; Fakultätszugehörigkeit: Wilks $\lambda$ $=.920, F(12,5368)=14.378, p<.001, \eta^{2}=.03$; Studienabschluss: Wilks $\lambda=.929, F(6$, $3920)=24.49, p<.001, \eta^{2}=.04 ;$ Kinder: Wilks $\lambda=.970, F(3,2033)=21.30, p<.001, \eta^{2}=$ .03; Migrationshintergrund: Wilks $\left.\lambda=.994, F(3,2033)=3.88, p=.009, \eta^{2}=.01\right)$.

Im Einzelnen zeigten sich folgende signifikanten Effekte: Für die selbsteingeschätzten Kompetenzen ergaben sich signifikante Effekte für das Geschlecht zugunsten der männlichen Studierenden, die höhere Werte berichteten $(M=5.16, S D=0.77)$ als ihre Kommilitoninnen $\left(M=4.65, S D=0.78 ; F(1,1802)=173.90, p<.001, \eta^{2}=.09\right)$. Ebenso zeigten sich für diese Variable signifikante Unterschiede in Abhängigkeit der Fakultätszugehörigkeit $\left(F(4,2036)=41.94, p<.001, \eta^{2}=.08\right)$. Klar am höchsten ausgeprägt waren die selbsteingeschätzten Fähigkeiten bei den Studierenden der 
Technischen Fakultät $(M=5.20, S D=0.71)$; am geringsten in der Philosophischen Fakultät mit Fachbereich Theologie $(M=4.65, S D=0.83)$. Schliesslich gab es Unterschiede hinsichtlich des angestrebten Studienabschlusses. Masterstudierende schätzten ihre Kompetenzen am höchsten ein $(M=5.13, S D=0.72)$, gefolgt von Bachelorstudierenden $(M=4.83, S D=0.80)$ und Studierenden mit Staatsexamen als Abschlussziel ( $M$ $\left.=4.58, S D=0.81, F(2,1965)=72.50, p<.001, \eta^{2}=.07\right)$. Studierende mit Kind schätzten ihre Fähigkeiten geringer ein $(M=4.59, S D=0.88)$ als Studierende ohne Kind $(M=4.82$, $\left.S D=0.81 ; F(1,2037)=5.77, p=.016, \eta^{2}=.00\right)$; der Effekt war jedoch von vernachlässigbarer Grösse.

Ein signifikanter Haupteffekt für die Variable zu betreuende Kinder zeigte sich bezüglich des Arbeitsplatzes. Studierende mit Kind hatten seltener Zugang zu einem ruhigen Arbeitsplatz $(M=0.69, S D=0.47)$ als Studierende ohne Kind $(M=0.93, S D=$ $\left.0.26 ; F(1,2037)=4.13, p<.001, \eta^{2}=.03\right)$. Ebenso hatten Studierende mit Migrationshintergrund seltener Zugang zu einem ruhigen Arbeitsplatz $(M=0.86 S D=0.34)$ als Studierende ohne Migrationshintergrund $(M=0.93, S D=0.26 ; F(1,2037)=10.71, p<$ $\left..001, \eta^{2}=.01\right)$.

Bezüglich der Nutzung von E-Learning Angeboten der Universität zeigt sich eine grosse Varianz zwischen den einzelnen Formaten. Während fast alle befragten Studierenden bereits Dateien für das Studium heruntergeladen hatten, haben rund $70 \%$ bereits einmal aufgezeichnete Lehrveranstaltungen genutzt, gefolgt von Online Kommunikation (Mail, Foren) für Studienzwecke, Online Selbsttest, Live-Votings und Online Lernmodulen. Etwas weniger als die Hälfte der befragten Studierenden hatte darüber hinaus bereits weitere online-gestützte Lernangebote in Anspruch genommen. Mit $4 \%$ hatten auffallend wenige Studierende an online gestreamten Lehrveranstaltungen teilgenommen. Weiterhin gaben $3 \%$ der Studierenden an, noch keine E-Learning Tools genutzt zu haben.

\begin{tabular}{|l|c|c|c|c|r|}
\hline \multicolumn{7}{|l|}{} & $\begin{array}{c}\text { vor Semes- } \\
\text { terstart }\end{array}$ & $\begin{array}{c}\text { zur Semes- } \\
\text { termitte }\end{array}$ & & \\
\cline { 2 - 6 } & $M(S D)$ & $M(S D)$ & $t(2036)$ & $p$ & $d$ \\
\hline Medienausstattung [0-1] & $0.92(0.27)$ & $0.94(0.23)$ & 3.81 & $<.001$ & 0.09 \\
\hline Ruhiger Arbeitsplatz & $1.00(0.07)$ & $1.00(0.04)$ & 1.73 & .083 & 0.05 \\
\hline Digitales Endgerät & & & & & \\
\hline Mediennutzung [1-5] & $4.59(0.67)$ & $4.78(0.46)$ & 12.79 & $<.001$ & 0.32 \\
\hline Digitales Endgerät & $4.87(0.69)$ & $4.86(0.70)$ & -1.03 & .304 & -0.02 \\
\hline Smartphone & $4.96(0.29)$ & $4.97(0.28)$ & 1.12 & .269 & 0.03 \\
\hline Internet & $4.81(0.81)$ & $5.01(0.75)$ & 18.73 & $<.001$ & 0.26 \\
\hline Digitale Kompetenzen [1-6] &
\end{tabular}




\begin{tabular}{|l|c|c|c|c|r|}
\hline \multicolumn{7}{|l|}{ E-Learning Tools [0-1] } \\
\hline Herunterladbare Dateien & $0.95(0.22)$ & $0.98(0.15)$ & 5.19 & $<.001$ & 0.15 \\
\hline $\begin{array}{l}\text { Lehrveranstaltungsaufzeich- } \\
\text { nungen }\end{array}$ & $0.69(0.46)$ & $0.88(0.32)$ & 17.76 & $<.001$ & 0.49 \\
\hline Online-Kommunikation & $0.64(0.48)$ & $0.79(0.41)$ & 12.17 & $<.001$ & 0.33 \\
\hline Online-Selbsttests & $0.55(0.50)$ & $0.59(0.49)$ & 3.27 & $<.001$ & 0.08 \\
\hline Live-Votings o.ä. & $0.54(0.50)$ & $0.58(0.49)$ & 3.43 & $<.001$ & 0.09 \\
\hline Online-Lernmodule & $0.52(0.50)$ & $0.67(0.47)$ & 12.21 & $<.001$ & 0.31 \\
\hline $\begin{array}{l}\text { Andere online-gestützte Lern- } \\
\text { angebote }\end{array}$ & $0.39(0.49)$ & $0.43(0.50)$ & 4.23 & $<.001$ & 0.09 \\
\hline Live-Streams & $0.04(0.20)$ & $0.70(0.46)$ & 60.48 & $<.001$ & 1.90 \\
\hline Keine & $0.03(0.17)$ & $0.00(0.05)$ & -6.72 & $<.001$ & -0.21 \\
\hline
\end{tabular}

Tab. 2.: Medienausstattung und Medien und E-Learning Tool Nutzung vor und während des Corona-Semesters. Anmerkung: Medienausstattung und Nutzung von E-Learning Tools wurden im nein/ja Format erfasst; die Mediennutzung wurde anhand einer Häufigkeitsskala (nie bis täglich) und digitale Kompetenzen mit einer 6-stufigen Likertskala (stimmt gar nicht bis stimmt genau) gemessen.

\subsection{Veränderungen im Corona-Semester}

Zur Abbildung von Veränderungen der Mediennutzung vom Semesterstart zur Semestermitte (F2) wurden gepaarte $t$-Tests durchgeführt (vgl. Tabelle 2). Zur Semestermitte nahm der Zugang zu einem ruhigen Arbeitsplatz zu, was angesichts der Schliessung von Bibliotheken, PC-Pools und Lernräumen darauf zurückzuführen sein dürfte, dass die Studierenden ihre häusliche Lernsituation verbessert hatten. Die Mediennutzung nahm von Semesterstart zur Semestermitte hin signifikant zu. Dies scheint mit studentischen Lernaktivitäten zusammenzuhängen, da im selben Zeitraum keine signifikante Steigerung bei der Smartphone- oder Internetnutzung zu verzeichnen war (vgl. Tabelle 2).

Im Semesterverlauf nahm die Nutzung aller institutionell angebotenen und im Survey abgefragten E-Learning-Tools signifikant zu. Der Anteil der Studierenden, die keine E-Learning Tools nutzen, sank auf $0 \%$ ab. Die grössten Steigerungen liessen sich bei den Lehrveranstaltungsaufzeichnungen (mittlere Effektstärke) und den LiveStreams (hohe Effektstärke) verzeichnen. Sehr schwach fielen die Zunahmen bei den Online-Selbsttests, Live-Votings und weiteren online-gestützten Lernangeboten aus. Neben einem gesteigerten Nutzungsverhalten hinsichtlich digitaler Lern- und Lehrmedien und -formate liess sich weiterhin eine Steigerung der selbstwahrgenommenen Fähigkeiten im Umgang mit digitalen Medien nachweisen. Dies galt für beide Subskalen mit jeweils grossen Effektstärken. 


\subsection{Unterschiedliche Nutzung von E-Learning Angeboten}

Exemplarisch für vier ausgewählte E-Learning Angebote, die in der Mitte des CoronaSemesters häufig verwendet wurden und Varianz in der Nutzung aufwiesen (Lehrveranstaltungsaufzeichnungen, Online-Kommunikation, Online-Lernmodule und LiveStreams), wurden Unterschiede hinsichtlich verschiedener Gruppen (Geschlecht weiblich vs. männlich, Fakultät, angestrebter Studienabschluss BA vs. MA, Studierende mit und ohne Kind und mit und ohne Migrationshintergrund) geprüft (F3); vgl. Tabelle 3 bzw. Abbildung 1. Die Ergebnisse der multivarianten Varianzanalysen erbrachten keine signifikanten Geschlechtsunterschiede (Wilks $\lambda=.993, F(8,3602)=$ $1.58, p=.130$ ), einen schwachen signifikanten Effekt für den Studienabschluss (Wilks $\left.\lambda=.991, F(4,1220)=2.81, p=.025, \eta^{2}=.01\right)$, der ausschliesslich auf die Nutzung von Livestreams zurückführbar ist und von vernachlässigbarer Grösse ist $(F(1,1223)$ $=5.19, p=.023, \eta^{2}=.004$, Bachelor: $n=734, M=0.67, S D=0.57$; Master: $n=491, M=$ $.74, S D=.44)$.

Unterschiede hinsichtlich des Migrationshintergrundes (Wilks $\lambda=.993, F(4,1799)$ $\left.=2.99, p=.018, \eta^{2}=.01\right)$ zeigen sich bei der Nutzung von Veranstaltungsaufzeichnungen, welche häufiger von Studierenden ohne Migrationshintergrund genutzt werden $(M=0.89, S D=0.31)$ als von Studierenden mit Migrationshintergrund $(M=0.83, S D=$ $\left.0.38 ; F(1,1802)=6.04, p=.014, \eta^{2}=.00\right)$. Auch hier ist die Effektstärke jedoch sehr klein. Keine signifikanten Nutzungsunterschiede zeigen sich in Abhängigkeit der familiären Situation (Wilks $\lambda=.996, F(4,1799)=1.58, p=.018, \eta^{2}=.00$ ). Deutlichere Effekte zeigen sich in Abhängigkeit der Fakultät (Wilks $\lambda=.860, F(16,6196)=19.57, p$ $\left.<.001, \eta^{2}=.04\right)$. In Tabelle 3 und Abbildung 1 sind die Unterschiede dargestellt.

\begin{tabular}{|l|c|c|c|}
\hline E-Learning Angebot & $F(4,2036)$ & $p$ & $\eta^{2}$ \\
\hline Lehrveranstaltungsaufzeichnungen & 35.832 & $<.001$ & .066 \\
\hline Live-Streams & 22.744 & $<.001$ & .043 \\
\hline Online-Lernmodule & 11.089 & $<.001$ & .021 \\
\hline Online-Kommunikation & 13.506 & $<.001$ & .026 \\
\hline
\end{tabular}

Tab. 3.: Nutzung der verschiedenen E-Learning Angebote in Abhängigkeit von der Fakultätszugehörigkeit.

Über alle Fakultäten hinweg wurde zur Semestermitte eine Kombination an verschiedenen E-Learning Angeboten genutzt. Diese scheint sich allerdings zwischen den Fakultäten zu unterscheiden. Während Lehrveranstaltungsaufzeichnungen vor allem in der Rechts- und Wirtschaftswissenschaftlichen Fakultät sowie der Medizinischen Fakultät genutzt wurden, wurden Live-Streams am häufigsten in der Naturwissenschaftlichen und Technischen Fakultät wahrgenommen, welche wiederum vergleichsweise selten Online-Lernmodule nutzten. Online Kommunikation wurde mit Abstand am häufigsten in der Philosophischen Fakultät genutzt. 


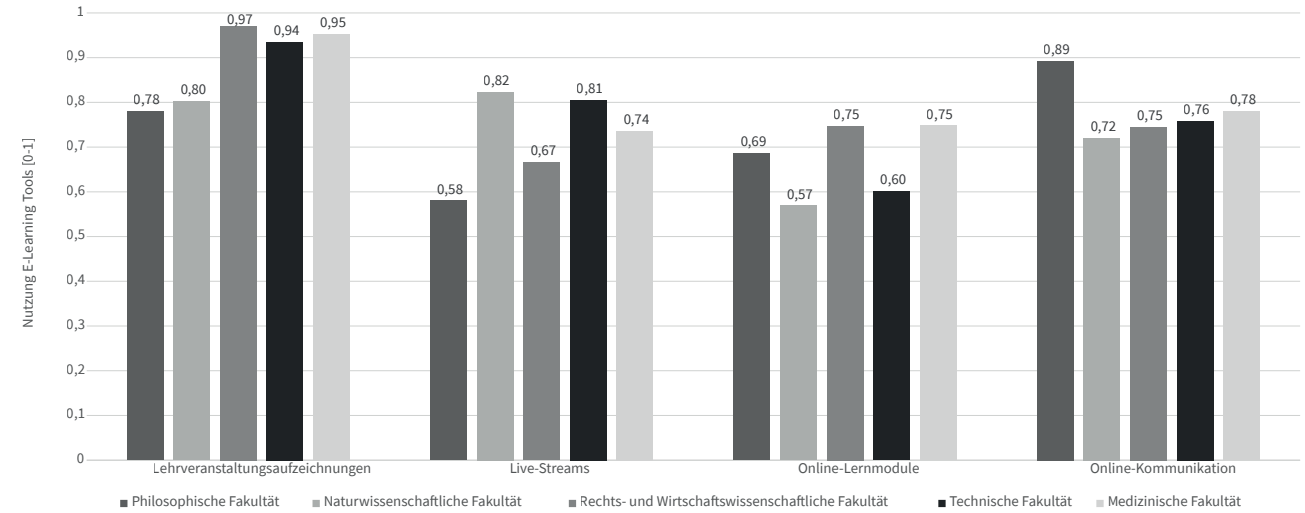

Abb. 1.: Nutzung der verschiedenen E-Learning Angebote in Abhängigkeit der Fakultätszugehörigkeit.

\section{Diskussion}

\subsection{Ausgangslage}

Die Nutzung institutionell bereitgestellter Formate und digitaler Lernelemente setzt eine Mindestausstattung an technischer Infrastruktur voraus - Hardware und Internetzugang - die im Falle der befragten Studierenden mit Zugang zu mindestens einem Gerät überwiegend gegeben war, zu einem kleineren Teil jedoch auch nicht. Karapanos et al. (2021) weisen in ihrer Studie darauf hin, dass Studierende ergänzende Hardware wie Mikrofone und Kameras zu Beginn des Semesters häufig nicht zur Verfügung hatten. In der vorliegenden Studie wurde diese Ausstattung allerdings nicht mit abgefragt. Im Sinne einer lernförderlichen Arbeitsumgebung kann konstatiert werden, dass Studierende mit Kind und Studierende mit Migrationshintergrund seltener einen ruhigen Arbeitsplatz zur Verfügung hatten, wobei gerade Ruhe ein Charakteristikum ist, dem Studierende Bedeutung zumessen (Beckers, van der Voordt, und Dewulf 2016). Unter der Perspektive von digital divides und digitaler Ungleichheit (Kutscher 2019; Verständig, Klein, und Iske 2016) verweisen quantitative Bestimmungen einerseits auf einen nur äusserst geringen Anteil an Studierenden, die nicht partizipieren konnten - eine stärker individuumszentrierte Betrachtung würde andererseits die personenbezogenen Auswirkungen deutlicher vor Augen führen und Konsequenzen für Partizipations- und Bildungsprozesse der Betroffenen aufzeigen können (Breitenbach 2021).

Die selbsteingeschätzten digitalen Kompetenzen der Studierenden sind in verschiedenen Studierendengruppen unterschiedlich stark ausgeprägt, wobei die Studierenden der Technischen Fakultät ihre Kompetenz am höchsten einschätzten, männliche Studierende höher als weibliche und fortgeschrittene Studierende höher 
als weniger fortgeschrittene. Diese Ergebnisse korrespondieren weitestgehend mit früherer Forschung (Senkbeil, Ihme, und Schöber 2019, 2020). Der Grossteil der Studierenden hatte auch bereits vor dem digitalen Semester Erfahrung mit unterschiedlichen E-Learning Tools im Studium, was vergleichbar dem heterogenen Stand an anderen Hochschulen ist (Arndt, Ladwig, und Knutzen 2020) ist.

\subsection{Veränderungen im Corona-Semester}

Das Sommersemester 2020 stand an Hochschulen unter der grundsätzlichen Bedingung, Lehre flächendeckend digital anzubieten. Aus dieser Situation heraus ist die signifikante Zunahme in der Nutzung von E-Learning-Tools heraus leicht nachvollziehbar, da eine Nichtnutzung faktisch eine Nichtteilnahme an Lehrveranstaltungen bedeutet hätte. In einer kritischen Perspektive kann hier argumentiert werden, dass Studierende mit einer extern gesetzten Selbstverständlichkeit (vgl. Aksoy und Neuberger 2020, 34) der Nutzungserwartung konfrontiert waren - die durch den Umstand der Pandemie über die Institutionen vermittelt wurde. Folgt man dem von Aksoy und Neuberger (2020) skizzierten Medienhandeln Studierender als «Erschließung von Studium, Wissenschaft und Universität mit und in digitalen Medien begriffen» (23-24), so ermöglicht die vorliegende Studie vor allem die Betrachtung der studentischen «Antwort auf gegebene Strukturen» (28) und weniger die Rekonstruktion des Eigensinns, den Studierende der (Nicht)Nutzung digitaler Medien im Studium zuschreiben. Nichtsdestotrotz erlauben die Ergebnisse einen Überblick über den grundlegenden Verlauf der Mediennutzung über das Sommersemester 2020 hinweg.

Die Nutzungszunahme zeigt auch, dass über die Fakultäten hinweg eine Mischung aus verschiedenen Tools eingesetzt wurde. Im Unterschied zu den Ergebnissen der Studie von Karapanos u. a. (2021), in der Studierende vor allem Lernmanagementsysteme, textbasierte Medien und Videokonferenzen als vorherrschende Technologien benannten, so sind es im Falle der hier untersuchten Universität vor allem Lehrveranstaltungsaufzeichnungen und Live-Streams, bei denen die Nutzung stark ansteigt - was im zeitlichen Vergleich mit vorherigen Semesters aber leicht erklärbar ist, da damit im Sommersemester 2020 Präsenzen im Hörsaal substituiert wurden.

Die Tatsache, dass Studierende ihre digitalen Kompetenzen zu Semesterbeginn als gut einschätzten und diese im Verlauf sogar noch aus subjektiver Sicht zunahmen, spiegelt die Ergebnisse der Befragung von Winde et al. (2020) wider, nach der diese kaum als Herausforderung seitens der Studierenden benannt wurden. Auch die Übersichtsarbeit von Arndt et al. (2020) zeigt ein ähnliches Bild, so dass hier eventuell auch die besondere Situation der Pandemie zum Tragen kommt. Für ein vertieftes Bild wäre es bei Studien dieser Art interessant, neben den Selbsteinschätzungen die Kompetenzen der Studierenden auch mittels Ausführung von Aufgaben zu erfassen (vgl. Senkbeil et al. 2019). 


\subsection{Unterschiedliche Nutzung von E-Learning Angeboten}

Die Ergebnisse der vorliegenden Befragungen zeigen auch auf, dass alle erfassten digitalen Formate und E-Learning Tools über die Fakultäten verteilt in den Lehrveranstaltungen eingesetzt wurden ( $M>50 \%)$. Gleichzeitig wurden in keiner Fakultät alle Formate angeboten, was einerseits durch die fakultäts- und fachspezifische Bevorzugung einzelner Formate zu erklären ist (Bond et al. 2020): So sind in der Philosophischen Fakultät mit diskurs- und diskussionsintensiven Disziplinen Formen der online Kommunikation sehr häufig eingesetzt, während Lehrveranstaltungsaufzeichnungen vor allem in der Rechts- und Wirtschaftswissenschaftlichen Fakultät sowie der Medizinischen Fakultät genutzt wurden. Zu bedenken ist hierbei auch, wie stark die Fakultäten bereits vor der Pandemie auf digitale Formate setzten (Händel, Bedenlier et al. 2020), wie dies bestehende und notwendigerweise aufgewandten Ressourcen beeinflusste und wie sich grundsätzlich disziplinäre Besonderheiten auf die Integration digitaler Medien in die Lehre auswirken (Mercader und Gairín 2020). Deutliche Hinweise auf eine unterschiedliche Nutzung von E-Learning Angeboten auf Grund weiterer soziodemografischer Charakteristika liessen sich in der vorliegenden Untersuchung nicht finden. Ergänzend zu der studentischen Nutzung der bereitgestellten E-Learning Angebote ist für die betreffende Institution festzuhalten, dass Lehrende, die erstmalig intensiv digitale Lehrformate gestaltet haben, als zukünftiges Ziel die Erhöhung der Interaktion in ihren Veranstaltungen angaben (Bedenlier et al. 2020), so dass sich dies perspektivisch auf das Angebot auswirken dürfte.

Im Rückbezug auf studentische Nutzung von E-Learning Tools im institutionalisierten akademischen Fächerkontext bedeutet die unterschiedliche Präferenz der Fakultäten für den Einsatz bestimmter Formate, dass Studierende eines Fachs vor allem mit diesen konfrontiert wurden - was wiederum nicht notwendigerweise ihren persönlichen Vorstellungen oder Zugängen zu Inhalten entsprechen muss (Persike und Friedrich 2016). Durch das bestehende Angebot wird ihr Nutzungsverhalten zu einem gewissen Grad gesteuert und sie sind hier vor allem als Rezipierende zu sehen. Wenngleich die besondere Situation der Pandemie als Kontext für das Handeln der involvierten Personen, Lehrende wie Lernende, zentral berücksichtigt werden muss, so zieht dies langfristig auch mediendidaktische Fragestellungen und Herausforderungen nach sich. Während das digitale Lehrformat in der Pandemie vor allem auf die Ermöglichung eines ortsunabhängigen Studiums abzielte, ist zukünftig stärker zu darauf achten, wie der Einsatz digitaler Medien in der Lehre genutzt werden kann, um Kompetenzen von Studierenden gezielt zu fördern. Während in der vorliegenden Studie die Nutzung vs. Nicht-Nutzung im Vordergrund stand, sind in künftigen Untersuchungen insbesondere auch die Intensität und die Qualität der Nutzung zu berücksichtigen. Die Integration neuer Medien in der Lehre knüpft einerseits an individuellen Vorerfahrungen bei den Studierenden an, sie schafft aber auch neue Situationen, in denen der Umgang mit neuen Medien geübt und auch neue Anwendungen 
und Fertigkeiten erlernt werden können. Wenn Studierende beispielsweise eine Präsentation anfertigen, üben sie auch computerunterstützte Präsentationstechniken und wenn sie an einem Peer-Assessment teilnehmen, lernen sie auch Formen computerunterstützter Beratung und Beurteilung kennen. Die unterschiedlichen Schwierigkeitsstufen dieser Tätigkeiten lassen sich didaktisch so arrangieren, dass sie von einfacheren zu komplexeren Aktionen führen und letztlich einem mediendidaktischen Curriculum folgen. Dieses Prinzip, das sich in der Mediendidaktik bei Kron und Sofos (2003) unter dem Stichwort «curricularer Transformationsprozess» wiederfindet, lässt sich in grössere Zusammenhänge einbringen, z. B. in den des erziehungswissenschaftlichen Grundstudiums oder den der drei Phasen der Lehrerbildung (Kammerl 2010). Die Grundidee dabei ist, dass auch in Lehrveranstaltungen, die keine expliziten Medienpädagogik-Veranstaltungen sind, Medien so verwendet werden, dass sie den Erwerb medienpädagogischer Kompetenzen ermöglichen, die für das Handeln in zukünftigen Handlungspraxen bedeutsam erscheinen. Gepaart mit inhaltlich ausgerichteten Lehrveranstaltungen zum Lernen mit und über Medien kann so, beispielsweise mit Hilfe einer abgestimmten Matrixstruktur, die Verankerung von Medienbildung vor allem auch in den Lehramtsstudiengängen vorangetrieben werden (Goetz und Kortenkamp 2019).

\section{Studienlimitationen und Ausblick}

\subsection{Limitationen}

Die Studie unterliegt verschiedenen Limitationen, die einerseits methodischer Art und andererseits der besonderen Situation der Pandemie geschuldet sind.

Bei den Teilnehmenden handelt es sich um eine selbstselektierte Gruppe, die einerseits die grundsätzlich nötige infrastrukturelle Ausstattung für die Teilnahme an einer Online-Befragung hatte, und andererseits die institutionell bereitgestellten Medien und Formate genutzt hat. Die Perspektive derjenigen Studierenden, die nicht zu dieser Gruppe gehören, ist somit in dieser Studie nicht abgebildet. Da die Befragung den studienbezogenen Kontext fokussierte, wurde der Bereich der informellen, privaten Mediennutzung ausgespart - obwohl anekdotische Belege vorliegen, die zeigen, dass bspw. Messengerdienste oder soziale Netzwerke eine Doppelfunktion an der Schnittstelle von Studium und Privatkommunikation einnehmen.

Eine weitere Limitation ergibt sich durch den methodischen Zugang, der in einer Linie mit vorherigen Mediennutzungsstudien steht und einen klar umgrenzten thematischen Bereich untersucht - und dies vor allem auch unter einer institutionellen Perspektive. Die Feststellung von Hofhues et al. $(2020,12)$ trifft grundsätzlich auch auf die vorliegende Studie zu: 
"Sie [bestehende Mediennutzungsstudien, d. Autoren] zeigen unter Rückgriff auf das quantitative Paradigma auf, in welchem Umfang Medien in der Universität genutzt werden und welche Implikationen sich hieraus beispielsweise für Serviceangebote und technische Infrastrukturen der Universität ergeben können. Gleichwohl hinterlassen sie Forschungslücken, indem deutlich weniger über konkrete Formen und Situationen studentischer Medienaneignung bekannt wird».

Besonderer Berücksichtigung bedarf die Tatsache, dass das Sommersemester 2020 geprägt war von der Unmittelbarkeit der didaktisch-technischen Umgestaltung der gesamten Lehre in digitale Formate und darum eine Ausnahmesituation untersucht wurde, die Studierende und Lehrende nicht zuletzt auch in einer sozial-emotional herausfordernden Lage vorfand (Händel, Stephan et al. 2020). Hinsichtlich der hier aufgezeigten Limitationen kann in zukünftiger Forschung ein stärker qualitativ ausgerichteter Zugang diesen begegnen, um so beispielsweise Facetten der digitalen Lehre unter Pandemiebedingungen noch stärker individualisiert zu betrachten und damit eine vertiefte Analyse zu ermöglichen (z. B. Autor:innengruppe AEDiL 2021).

\subsection{Ausblick}

Die vorliegende Studie ermöglicht einen ersten Einblick in das Medien- und E-Learning Tool Nutzungsverhalten von Studierenden an einer Volluniversität im digitalen Sommersemester 2020. Während die Studie methodisch und thematisch in einer Reihe steht mit anderen quantitativen Untersuchungen vor und während der Pandemie zu studentischer Mediennutzung, so zeigt sie auf, dass Studierende einen Zuwachs ihrer digitalen Kompetenzen über den Semesterverlauf feststellten, E-Learning Tools fakultätsspezifisch für Lehr- und Lernzwecke eingesetzt werden und dass Studierende überwiegend gut für digitale Lehrangebote ausgestattet sind. Diese Ergebnisse stellen eine Grundlage für vertiefende Studien mit einem weiteren inhaltlichen Fokus auf studentische Nutzung von Medien und E-Learning Tools und studentische Medienkompetenz an der Schnittstelle von Studium und Privatleben dar. Solch ein Fokus würde es ermöglichen, einzelne Studierendengruppen sowie bestimmte Fächerkulturen bezüglich digital-vermittelter Lehre genauer zu untersuchen. Implikationen für die Ausgestaltung der Digitalisierung in der Hochschullehre können hinsichtlich der Diskussion ihrer Motive, der Vorbereitung der Lehrenden und der Berücksichtigung der studentischen Perspektive gezogen werden:

Inwieweit die aktuelle Voll-Digitalisierung hochschulischer Lehrangebote einen nachhaltigen Digitalisierungsschub bewirkt, ist zum jetzigen Zeitpunkt kaum zu beurteilen (Kerres 2020; Vallaster und Sageder 2020; Zawacki-Richter 2020). Während die bildungspolitischen Zielsetzungen und Bestrebungen - inklusive finanzieller 
Förderung von Lehrprojekten - in den letzten Jahren sicherlich einen Grundstein dafür gelegt haben, dass infrastrukturell und mediendidaktisch begleitet ein Wechsel von vornehmlich Präsenz auf reine Online-Lehre erfolgen konnte, so ist aktuell offen, inwiefern digitale Formate weiterhin bei den Studierenden ihre Akzeptanz behalten, wenn eine Rückkehr zur Präsenzlehre möglich ist. Sicherlich trägt die Flexibilisierung des Studiums durch Erhöhung der zeitlichen und örtlichen Unabhängigkeit bei bestimmten Studierendengruppen zu einer Offenheit für digitale Formen bei, es ist aber auch davon auszugehen, dass sich die Studierenden ganz bewusst für ein Präsenzstudium und nicht für eines der bereits vor der Pandemie existierenden OnlineAngebote entschieden haben. In den letzten Jahren war gerade in den «Massenfächern» zu beobachten, dass digitale Formate, wie z. B. MOOCs, nicht nur aus didaktischen Überlegungen oder studentischen Interessen heraus vorangetrieben wurden, sondern ökonomischen Strategien folgte (Schulmeister 2013). Welchen Zielen und wessen Interessen der zu erwartende Digitalisierungsschub nutzt, ist deshalb eine zunehmend wichtige Fragestellung.

Hinsichtlich der Ausstattung technischer Art und des Verfügens über digitale Kompetenzen, bzw. akademische Medienkompetenz, gilt es, ein mögliches Auseinanderdriften von digital kompetenten und nicht oder weniger kompetenten Studierenden und Lehrenden zu verhindern. Späteren Benachteiligungen kann so vorgebeugt werden (Senkbeil, Ihme, und Schöber 2020). Das bedeutet auch, Lehr-Lernangebote an der Hochschule so zu gestalten, dass der kompetente Umgang mit digitalen Medien und Formaten fachlich gebunden erlernt werden kann; hier bedarf es mediendidaktisch fundierter Interventionen, die auch institutionell unterstützt und vorangetrieben werden sollte. Weiterführende Studien und Praktiken in diesem Feld bedürfen dann jedoch auch der bewussten Auseinandersetzung mit studentischem medialen Handeln, welches weniger unter dem aktuellen Fokus auf Studierende als Reagierende und Rezipierende in einem pandemiedeterminierten Hochschulkontext steht, sondern auf die individuelle studentische Praktik (Steinhardt 2020) und deren «eigensinniges Handeln» (Hofhues et al. 2020) fokussiert.

Während einige Lehrende für die Gestaltung ihrer Veranstaltungen Potentiale in digitalen Medien erkannt haben und ihrem Einsatz positiv gegenüberstehen (Winde et al. 2020), so verweist der Aufruf zum Nichtsemester im Frühjahr 2020 auf einen nicht unerheblichen Teil an Lehrenden mit weiterhin kritischer Einstellung, zumindest was die Umsetzung von ad hoc Online-Lehre angeht. Arndt, Ladwig und Knutzen $(2020,32)$ resümieren hochschulübergreifend, dass zwischen $40 \%$ und $70 \%$ der Lehrenden angaben, vor dem Sommersemester 2020 kaum oder wenig Erfahrungen mit digitaler Lehre gemacht zu haben. Auch wenn dieser Anteil durch die vergangenen digitalen Semester zwangsläufig gesunken ist, so besteht weiterhin der Bedarf an Schulungen für Lehrende, um didaktisches und technisches Wissen weiterhin aufzubauen und zu stärken. 
Schliesslich stellen sich, vor allem auch im Kontext der zunehmenden Digitalisierung und des Einsatzes von digitalen Medien und Formaten in der Lehre - die auch nach CoViD-19 bleiben werden - Fragen nach dem Stellenwert von Lehre und dem damit verbundenen hochschulischen Selbstverständnis. Während ein «weiter wie bisher» mit dem Anhalten der Pandemie unwahrscheinlicher wird, so bleibt die Gestaltung der Digitalisierung in der Hochschullehre ein Prozess, der zwischen Lernenden, Lehrenden und der Institution ausgehandelt wird. Impulse aus der Lehre unter pandemischen Bedingungen (z. B. Autor:innengruppe AEDiL) können hierbei einen wertvollen Beitrag leisten.

\section{Literatur}

Aksoy, Filiz, und Neuberger, Olga. 2020. «Eigensinniges Medienhandeln Studierender?». In Studierende - Medien - Universität, herausgegeben von Sandra Hofhues, Mandy Schiefner-Rohs, Sandra Assmann und Taiga Brahm, 23-40. Münster: Waxmann. https://doi. org/10.25656/01:20504.

Arndt, Christiane, Tina Ladwig, und Sönke Knutzen. 2020. «Zwischen Neugier und Verunsicherung: interne Hochschulbefragungen von Studierenden und Lehrenden im virtuellen Sommersemester 2020: Ergebnisse einer qualitativen Inhaltsanalyse». November. https://doi. org/10.15480/882.3090.

Autor:innengruppe AEDiL. 2021. «Corona-Semester reflektiert. Einblicke einer kollaborativen Autoethnographie». Bielefeld: wbv. https://doi.org/10.3278/6004820w.

Beckers, Ronald, Theo van der Voordt, und Geert Dewulf. 2016. «Learning Space Preferences of Higher Education Students». Building and Environment 104 (August): 243-52. https://doi. org/10.1016/j.buildenv.2016.05.013.

Bedenlier, Svenja, und Markus Deimann. 2020. «,Bildung' und ,Digitalisierung' im Spiegel von Digitalisierungsstrategien». Zeitschrift für Hochschulentwicklung 15 (1): 41-59. https://doi. org/10.3217/zfhe-15-01/03.

Bedenlier Svenja, Claudia Schmidt, Stefanie Gerl, Sonia Hetzner, Mona Schliebs, und Katja Sesselmann. 2020. «I can do this - better than I thought. Instrutcors' experiences of the digital summer term 2020». European Distance and E-Learning Network (EDEN) Proceedings 2020 Research Workshop, Lisbon 21-23 October. https://10.38069/edenconf-2020-rw0002.

Bond Melissa, Katja Buntins, Svenja Bedenlier, Olaf Zawacki-Richter, und Michael Kerres. 2020. «Mapping Research in Student Engagement and Educational Technology in Higher Education: A Systematic Evidence Map». International Journal of Educational Technology in Higher Education 17 (1): 2. https://doi.org/10.1186/s41239-019-0176-8. 
Bond, Melissa, Victoria I. Marín, Carina Dolch, Svenja Bedenlier, und Olaf Zawacki-Richter. 2018. «Digital Transformation in German Higher Education: Student and Teacher Perceptions and Usage of Digital Media». International Journal of Educational Technology in Higher Education 15 (1): 48. https://doi.org/10.1186/s41239-018-0130-1.

Breitenbach, Andrea. 2021. Digitale Lehre in Zeiten von Covid-19: Risiken und Chancen. Marburg. https://doi.org/10.25656/01:21274.

Carretero, Stephanie, Riina Vuorikari, und Yves Punie. 2017. «DigComp 2.1: The Digital Competence Framework for Citizens with eight proficiency levels and examples of use». https:// doi.org/10.2760/38842.

«Das Sommersemester 2020 muss ein ,Nichtsemester' werden - Ein offener Brief aus Forschung und Lehre». 2020. https://www.nichtsemester.de/cbxpetition/offener-brief/index. html\#unterschriften.

Daumiller, Martin, Raven Rinas, Julia Hein, Stefan Janke, Oliver Dickhäuser, und Markus Dresel. 2021. «Shifting from Face-to-Face to Online Teaching during COVID-19: The Role of University Faculty Achievement Goals for Attitudes towards This Sudden Change, and Their Relevance for Burnout/Engagement and Student Evaluations of Teaching Quality». Computers in Human Behavior 118 (Mai): 106677. https://doi.org/10.1016/j.chb.2020.106677.

Dolch, Carina, und Olaf Zawacki-Richter. 2018. «Are students getting used to Learning Technology? Changing media usage patterns of traditional and non-traditional students in higher education». Research in Learning Technology 26 (0). https://doi.org/10.25304/rlt.v26.2038.

FernUniversität in Hagen. 2020. «Lernen neu denken. Das Hagener Manifest zu New Learning». https://www.fernuni-hagen.de/universitaet/hagener-manifest/das-hagener-manifest.sht$\mathrm{ml}$.

Froebus, Nicole, und Desirée Bender. 2019. Abschlussbericht zur FAU-Studierendenbefragung FAU-St 2019. Friedrich-Alexander-Universität Erlangen-Nürnberg. https://nbn-resolving. org/urn:nbn:de:bvb:29-opus4-140454.

Gidion, Gerd, und Michael Weyrich. 2017. Mediale Hochschul-Perspektiven 2020 in Baden-Württemberg. Empirische Untersuchung im Rahmen der Allianz „Forward IT“. Karlsruhe: KIT Scientific Publishing. http://doi.org/10.5445/ksp/1000064688.

Gilch, Harald, Anna Sophia Beise, René Krempkow, Marko Müller, Friedrich Stratmann, und Klaus Wannemacher. 2019. «Digitalisierung der Hochschulen. Ergebnisse einer Schwerpunktstudie für die Expertenkommission Forschung und Innovation». Studien zum deutschen Innovationssystem Nr. 14-2019. HIS-Institut für Hochschulentwicklung. https://hishe.de/fileadmin/user_upload/Publikationen/Externe_Publikationen/StuDIS_14_2019.pdf. 
Goetz, Ilka, und Kortenkamp, Ulrich. 2019. «Die Umsetzung der Medienbildung in der Lehrerinnen-und Lehrerbildung an der Universität Potsdam - initiiert durch ein fachdidaktisches Entwicklungsvorhaben». In Digitale Medien in der Grundschullehrerbildung. Erfahrungen aus dem Projekt di-leg-SL. Schriftenreihe Medienpädagogik interdisziplinär, Band 12., herausgegeben von Thorsten Junge und Horst Niesyto, 397-408. München: Verlag kopaed. https://www.ph-ludwigsburg.de/fileadmin/phlb/hochschule/fakultaet1/erziehungswissenschaft/medienpaedagogik/12-Abgeschlossene_Projekte/dileg_SL/dileg-SL-2019-Goetz_Kortenkamp_-_Umsetzung_der_Medienbildung_in_der_Lehrerbildung.pdf.

Grosch, Michael, und Gerd Gidion. 2011. Mediennutzungsgewohnheiten im Wandel: Ergebnisse einer Befragung zur studiumsbezogenen Mediennutzung. Karlsruhe: KIT Scientific Publishing. https://doi.org/10.5445/KSP/1000022524.

Händel, Marion, Melanie Stephan, Michaela Gläser-Zikuda, Bärbel Kopp, Svenja Bedenlier, und Albert Ziegler. 2020. "Digital Readiness and Its Effects on Higher Education Students' Socio-Emotional Perceptions in the Context of the COVID-19 Pandemic». Journal of Research on Technology in Education, November, 1-13. https://doi.org/10.1080/15391523.2020.184 6147.

Händel, Marion, Svenja Bedenlier, Michaela Gläser-Zikuda, Rudolf Kammerl, Bärbel Kopp, und Albert Ziegler. 2020. «Do Students Have the Means to Learn During the Coronavirus Pandemic? Student Demands for Distance Learning in a Suddenly Digital Landscape.» PsyArXiv. July 1. https://doi.org/10.31234/osf.io/5ngm9.

Hochschulforum Digitalisierung. 2015. Diskussionspapier- 20 Thesen zur Digitalisierung der Hochschulbildung. Bd. 14. Berlin: Hochschulforum Digitalisierung. https://hochschulforumdigitalisierung.de/sites/default/files/dateien/HFD-Thesenpapier_Sep2015.pdf.

Hodges, Charles, Stephanie Moore, Barb Lockee, Torrey Trust, und Aaron Bond. 2020. "The difference between emergency remote teaching and online learning». EDUCAUSEreview, März. https://er.educause.edu/articles/2020/3/the-difference-between-emergency-remote-teaching-and-online-learning.

Hofhues, Sandra, Mandy Schiefner-Rohs, Sandra Assmann, und Taiga Brahm, Hrsg. 2020. Studierende - Medien - Universität. Waxmann Verlag GmbH. https://doi. org/10.31244/9783830990499.

Hong, Ah Jeong, und Hye Jeong Kim. 2018. «College Students’ Digital Readiness for Academic Engagement (DRAE) Scale: Scale Development and Validation». The Asia-Pacific Education Researcher 27 (4): 303-12. https://doi.org/10.1007/s40299-018-0387-0.

Jörissen, Benjamin. 2011. «Medienbildung〉- Begriffsverständnisse und Reichweiten». In Medienbildung und Medienkompetenz. Beiträge zu Schlüsselbegriffen der Medienpädagogik, herausgegeben von Heinz Moser, Petra Grell, und Horst Niesyto, 211-35. München: kopaed. https://doi.org/10.21240/mpaed/20/2011.09.20.X.

Kammerl, Rudolf. 2010. «Theoretische und empirische Aspekte zur Integration von E-LearningDiensten an Hochschulen». In E-Learning in Hochschule und Weiterbildung. Einsatzchancen und Erfahrungen, herausgegeben von Roland Holten und Dieter Nittel, 19-33. Bielefeld: Bertelsmann 
Karapanos, Marios, Robert Pelz, Patrick Hawlitschek, und Heinz-Werner Wollersheim. 2021. «Hochschullehre im Pandemiebetrieb: Wie Studierende in Sachsen das digitale Sommersemester erlebten». MedienPädagogik: Zeitschrift für Theorie und Praxis der Medienbildung 40 (Januar): 1-24. https://doi.org/10.21240/mpaed/40/2021.01.28.X.

Kerres, Michael. 2020. «Against All Odds: Education in Germany Coping with Covid-19». Postdigital Science and Education, Mai, s42438-020-00130-37. https://doi.org/10.1007/s42438020-00130-7.

Kreidl, Christian, und Ullrich Dittler. 2018. «Wo stehen wir? Ergebnisse einer umfassenden empirischen Studie zu Lernen und Unterricht an Hochschulen heute». In Hochschule der Zukunft, herausgegeben von Ullrich Dittler und Christian Kreidl, 35-62. Wiesbaden: Springer Fachmedien Wiesbaden. https://doi.org/10.1007/978-3-658-20403-7_3.

Kron, Friedrich, und Alivisos Sofos. 2003. Mediendidaktik. UTB. Reinhardt: München

Kultusministerkonferenz. 2019. «Empfehlungen zur Digitalisierung in der Hochschullehre. Beschluss der Kultusministerkonferenz vom 14.03.2019». Berlin/Bonn: Sekretariat der Ständigen Konferenz der Kultusminister der Länder in der Bundesrepublik Deutschland.

Kutscher, Nadia. 2019. «Digitale Ungleichheit als Herausforderung für Medienbildung». DDS Die Deutsche Schule, 111(4), 379-390. https://doi.org/10.31244/dds.2019.04.02.

Lei, Jing. 2009. «Digital natives as preservice teachers: What technology preparation Is needed?» Journal of Computing in Teacher Education 25 (3): 87-97. https://doi.org/10.1080/10 402454.2009.10784615.

Mercader, Cristina, und Joaquín Gairín. 2020. «University Teachers' Perception of Barriers to the Use of Digital Technologies: The Importance of the Academic Discipline». International Journal of Educational Technology in Higher Education 17 (1): 4. https://doi.org/10.1186/ s41239-020-0182-x.

Muilenburg, Lin Y., und Zane L. Berge. 2005. «Student Barriers to Online Learning: A Factor Analytic Study». Distance Education 26 (1): 29-48. https://doi.org/10.1080/01587910500081269.

Mulders, Miriam, und Sophia Krah. 2021. «Digitales Lernen während der Covid-19-Pandemie aus Sicht von Studierenden der Erziehungswissenschaften: Handlungsempfehlungen für die Digitalisierung von Hochschullehre». MedienPädagogik: Zeitschrift für Theorie und Praxis der Medienbildung 40 (Januar): 25-44. https://doi.org/10.21240/mpaed/40/2021.02.02.X.

Persike, Malte, und Julius-David Friedrich. 2016. «Lernen mit digitalen Medien aus Studierendenperspektive. Sonderauswertung aus dem CHE Hochschulranking für die deutschen Hochschulen». Arbeitspapier 17. Berlin: Hochschulforum Digitalisierung.

Prensky, Marc. 2001. «Digital Natives, Digital Immigrants Part 1». On the Horizon 9 (5): 1-6. https://doi.org/10.1108/10748120110424816.

Ruhr-Universität Bochum. o. J. «Digitalisierungsstrategie für die Lehre». Zugegriffen 22. Februar 2021. https://uni.ruhr-uni-bochum.de/de/digitalisierungsstrategie-fuer-die-lehre.

RWTH Aachen. o. J. «Digitalisierungsstrategie der Lehre». https://www.rwth-aachen.de/cms/ root/Studium/Lehre/ hjfu/Digitalisierungsstrategie-der-Lehre/. 
Schmid, Ulrich, Lutz Goertz, Sabine Radomski, Sabrina Thom, und Julia Behrens. 2017. Monitor Digitale Bildung. Die Hochschulen im digitalen Zeitalter. Gütersloh: Bertelsmann.

Schmölz, Alexander, Corinna Geppert, und Alessandro Barberi. 2020. «Digitale Kluft: Teilhabebarrieren für Studierende durch universitäres home learning?» Medienimpulse, Juni, https://doi.org/10.21243/MI-02-20-31.

Schulmeister, Rolf, Hrsg. 2013. MOOCs. Massive Open Online Courses. Offene Bildung oder Geschäftsmodell? Münster, New York. München Berlin: Waxmann,

Senkbeil, Martin, Jan Marten Ihme, und Christian Schöber. 2019. «Wie gut sind angehende und fortgeschrittene Studierende auf das Leben und Arbeiten in der digitalen Welt vorbereitet? Ergebnisse eines Standard Setting-Verfahrens zur Beschreibung von ICT-bezogenen Kompetenzniveaus». Zeitschrift für Erziehungswissenschaft 22 (6): 1359-84. https://doi. org/10.1007/s11618-019-00914-z.

Senkbeil, Martin, Jan Marten Ihme, und Christian Schöber. 2020. «Schulische Medienkompetenzförderung in einer digitalen Welt: Über welche digitalen Kompetenzen verfügen angehende Lehrkräfte?» Psychologie in Erziehung und Unterricht 67. https://doi.org/10.2378/ peu2020.art12d.

Skulmowski, Alexander, und Günter Daniel Rey. 2020. «Covid-19 as an Accelerator for Digitalization at a German University: Establishing Hybrid Campuses in Times of Crisis». Human Behavior and Emerging Technologies 2 (3): 212-16. https://doi.org/10.1002/hbe2.201.

Steffens, Yannic, Inga Lotta Schmitt, und Sandra Assmann. 2017. «Mediennutzung Studierender: Über den Umgang mit Medien in hochschulischen Kontexten. Systematisches Review nationaler und internationaler Studien zur Mediennutzung Studierender». Köln. https:// doi.org/10.13154/rub.106.95.

Steinhardt, Isabel. 2020. «Digitale Praktiken und das Studium.» SocArXiv. May 22. https://doi. org/10.31235/osf.io/rebh7.

Tulodziecki, Gerhard. 2011. «Zur Entstehung und Entwicklung zentraler Begriffe bei der pädagogischen Auseinandersetzung mit Medien». In Medienbildung und Medienkompetenz, herausgegeben von Heinz Moser, Petra Grell und Horst Niesyto, 11-40. München: Kopaed. https://doi.org/10.21240/mpaed/20/2011.09.11.X.

Vallaster, Christine und Sageder Martina. 2020. «Verändert Covid-19 die Akzeptanz virtueller Lehrformate in der Hochschulausbildung? Implikationen für die Hochschulentwicklung». Zeitschrift für Hochschulentwicklung 15 (4): 281-301. https://doi.org/10.3217/ZFHE-15$04 / 16$.

Vennemann, Mario, Knut Schwippert, Birgit Eickelmann, und Corinna Massek. 2019. "Computer- und informationsbezogene Kompetenzen von Schülerinnen und Schülern mit und ohne Migrationshintergrund im zweiten internationalen Vergleich». In ICILS 2018 \#Deutschland. Computer- und informationsbezogene Kompetenzen von Schülerinnen und Schülern im zweiten internationalen Vergleich und Kompetenzen im Bereich Computational Thinking, herausgegeben von Birgit Eickelmann, Wilfried Bos, Julia Gerick, Frank Goldhammer, Heike Schaumburg, Knut Schwippert, Martin Senkbeil, und Jan Vahrenhold, 335-65. Münster: Waxmann. https://doi.org/10.25656/01:18329. 
Verständig, Dan, Alexandra Klein, und Stefan Iske. 2016. «Zero-Level Digital Divide: neues Netz und neue Ungleichheiten.» SIEGEN: SOZIAL-Analysen, Berichte, Kontroversen (SI: SO), Nr. 1: 50-55. https://nbn-resolving.org/urn:nbn:de:hbz:467-11973.

Winde, Mathias, Said D. Werner, Barbara Gumbmann, und Solveigh Hieronimus. 2020. Hochschulen, Corona und jetzt? Wie Hochschulen vom Krisenmodus zu neuen Lehrstrategien für die digitale Welt gelangen. Herausgegeben von Stifterverband für die Deutsche Wissenschaft e.V. Future Skills - Diskussionspapier 4. Essen. https://www.stifterverband.org/medien/hochschulen-corona-und-jetzt.

Zawacki-Richter, Olaf. 2020. «Halb zog sie ihn, halb sank er hin... Covid-19 als Chance für die Digitalisierung von Studium und Lehre?». Das Hochschulwesen 68 (4+5).

Zawacki-Richter, Olaf, Carina Kramer, und Wolfgang Müskens. 2016. «Studiumsbezogene Mediennutzung im Wandel Querschnittdaten 2012 und 2015 im Vergleich». Schriftenreihe zum Bildungs- und Wissenschaftsmanagement 1. https://openjournal.uni-oldenburg.de/index. php/bildungsmanagement/article/view/101.

Zawacki-Richter, Olaf. 2015. «Zur Mediennutzung im Studium - unter besonderer Berücksichtigung heterogener Studierender». Zeitschrift für Erziehungswissenschaft 18 (3): 527-49. https://doi.org/10.1007/s11618-015-0618-6. 